\title{
Dronabinol for chemotherapy-induced nausea and vomiting unresponsive to antiemetics
}

This article was published in the following Dove Press journal:

Cancer Management and Research

12 May 2016

Number of times this article has been viewed

\author{
Megan Brafford May' \\ Ashley E Glode ${ }^{2}$ \\ 'Department of Pharmacy, Baptist \\ Health Lexington, Lexington, KY, USA; \\ ${ }^{2}$ Department of Clinical Pharmacy, \\ Skaggs School of Pharmacy and \\ Pharmaceutical Sciences, University of \\ Colorado Anschutz Medical Campus, \\ Aurora, CO, USA
}

\begin{abstract}
Chemotherapy-induced nausea and vomiting (CINV) is one of the most common symptoms feared by patients, but may be prevented or lessened with appropriate medications. Several antiemetic options exist to manage CINV. Corticosteroids, serotonin receptor antagonists, and neurokinin receptor antagonists are the classes most commonly used in the prevention of CINV. There are many alternative drug classes utilized for the prevention and management of CINV such as antihistamines, benzodiazepines, anticonvulsants, cannabinoids, and dopamine receptor antagonists. Medications belonging to these classes generally have lower efficacy and are associated with more adverse effects. They are also not as well studied compared to the aforementioned agents. This review will focus on dronabinol, a member of the cannabinoid class, and its role in CINV. Cannabis sativa L. (also known as marijuana) contains naturally occurring delta-9-tetrahydrocannibinol (delta-9-THC). The synthetic version of delta-9-THC is the active ingredient in dronabinol that makes dronabinol an orally active cannabinoid. Evidence for clinical efficacy of dronabinol will be analyzed in this review as monotherapy, in combination with ondansetron, and in combination with prochlorperazine.
\end{abstract}

Keywords: dronabinol, cannabinoids, antiemetic, chemotherapy-induced nausea and vomiting

\section{Introduction}

Chemotherapy-induced nausea and vomiting (CINV) is one of the most common symptoms feared by patients, but may be prevented or lessened with appropriate medications. ${ }^{1}$ CINV is categorized as acute, delayed, anticipatory, breakthrough, or refractory. ${ }^{2}$ Acute-onset CINV may occur within a few minutes to several hours after treatment administration and typically resolves within the first 24 hours. Risk factors for the development of this kind of nausea include female sex, age $<50$ years, environment of administration, lack of history of chronic alcoholism, history of motion sickness, previous incidence of nausea and vomiting, emetogenicity of agent(s) administered, dose of emetogenic agent(s), infusion rate, duration of therapy, number of cycles, and efficacy of preventive antiemetic regimen. Delayedonset CINV generally occurs in patients more than 24 hours after medication administration and may last 6-7 days. This type of nausea unfortunately tends to be more common, severe, and treatment resistant. Factors impacting the incidence of delayed CINV include dose and emetogenicity of chemotherapy agent(s), incidence of acute CINV, patient age, sex, and prophylactic antiemetics used. Anticipatory nausea and/or vomiting is considered a conditioned response and may occur after a negative prior experience with chemotherapy. This happens before a patient receives
Correspondence: Megan Brafford May Baptist Health Lexington, 1740 Nicholasville Road, Lexington, KY, 40503, USA

Email megan.may@bhsi.com 
their next cycle of treatment. This type of CINV is more common in younger patients due to the aggressive nature of treatment. Breakthrough emesis is considered vomiting that occurs even with prophylactic treatment and/or requires the addition of "rescue" antiemetics. Refractory emesis is classified as vomiting that occurs with subsequent treatment when prophylactic antiemetics and/or rescue medications have been ineffective in previous cycles.

CINV may have a significant negative impact on a patient's quality of life (QoL) as well as lead to increased indirect and direct costs. When a patient's QoL is impacted in a negative manner, it may result in poor compliance with future treatment. The development of CINV has been reported to have a significant impact on QoL in 55\%-60\% of chemotherapy cycles administered to patients. ${ }^{1}$ The intensity of CINV and duration of CINV were the most notable factors linked to a more significant influence on QoL. In a study analyzing cost associated with the development of CINV, it was noted that the average cost was nearly $\$ 800$ per patient for the first 5 days of the first cycle of chemotherapy. ${ }^{3}$ The cost analysis included overall direct and indirect costs: direct medical, missed work, productivity loss, and cost of any antiemetic medications taken on the day of treatment. Indirect costs were greater for patients with more severe CINV due to work absence for longer periods of time and reduced productivity. Additional undesirable effects of CINV to consider include metabolic imbalances, decline in self-care and functional ability, nutrient depletion, anorexia, worsening of the patient's performance status and mental status, wound dehiscence, esophageal tears, and withdrawal from potentially useful or curative treatment. ${ }^{2}$

It is reported that chemotherapy-induced vomiting can be prevented in more than two-thirds of patients if antiemetics are used correctly. ${ }^{1}$ Nausea remains difficult to prevent and manage and is typically more commonly reported than emesis: $54.9 \%$ versus $45.1 \%$ at any cycle. Several treatment guidelines exist published by reliable institutions providing recommendations to optimize CINV prevention and management, yet CINV remains an issue. In a recent report, despite prophylactic antiemetics, approximately $60 \%$ of patients who received moderately and highly emetogenic chemotherapy regimens still experienced some form of CINV; delayed being more common (58\% versus $34 \%$ ). ${ }^{3}$ Improvements in CINV management are needed.

\section{Current antiemetic treatment}

Several antiemetic options exist to manage CINV. Corticosteroids, serotonin receptor antagonists (5- $\left.\mathrm{HT}_{3} \mathrm{RAs}\right)$, and neurokinin receptor antagonists $\left(\mathrm{NK}_{1} \mathrm{RAs}\right)$ are the classes most commonly used in the prevention of CINV.

Corticosteroids have been utilized as an effective antiemetic for over 30 years, with dexamethasone being the most common agent. ${ }^{4}$ This class may be used for acute and delayed CINV and are effective when given in combination for prevention of CINV in moderately and highly emetogenic regimens. A meta-analysis of over 5,000 patients receiving highly or moderately emetogenic chemotherapy assessed the efficacy of dexamethasone as prophylaxis for CINV. In the majority of trials included, dexamethasone was given in combination with other antiemetics, such as 5-HT 3 RAs, or metoclopramide. Dexamethasone was determined to be superior to placebo or no treatment in terms of complete protection (no vomiting or retching) for both acute (odds ratio: $2.22,95 \%$ confidence interval: $1.89-2.60)$ and delayed (odds ratio: $2.04,95 \%$ confidence interval: 1.63-2.56) vomiting. Corticosteroids are usually well tolerated when used as a short-term antiemetic. Moderate-to-severe insomnia (45\%), gastrointestinal discomfort (27\%), agitation $(27 \%)$, increased appetite (19\%), weight gain (16\%), and acne (15\%) have been reported by patients taking dexamethasone for the prevention of delayed CINV.

Selective $5-\mathrm{HT}_{3} \mathrm{RAs}$ have been incorporated into the management of CINV for over the last 20 years. ${ }^{4}$ In the United States, the following agents are approved: dolasetron, granisetron, ondansetron, and palonosetron, with palonosetron having a significantly longer half-life, making it exceptionally useful in the prevention of delayed CINV. Various studies have shown this class of agents to be effective in the prevention of acute and delayed CINV. One meta-analysis including ten studies revealed an $8.2 \%$ absolute risk reduction compared to placebo for the development of delayed CINV. One of the more notable adverse effects of this class is the potential development of electrocardiogram abnormalities, including QT prolongation. Additional side effects reported with these medications include headache, constipation, and abdominal pain.

One of the newer classes of medications approved for the prevention of CINV is the $\mathrm{NK}_{1}$ RAs. Agents from this class should be administered in combination with dexamethasone and a 5-HT 3 RA to prevent acute and delayed CINV associated with moderately and highly emetogenic chemotherapy. ${ }^{2}$ A meta-analysis including nearly 9,000 patients receiving moderately and highly emetogenic chemotherapy experienced a significant improvement in CINV in the acute, delayed, and overall phases $(P<0.001)$ when $\mathrm{NK}_{1}$ RAs were added to $5-\mathrm{HT}_{3}$ RAs and corticosteroids. ${ }^{4}$ Medications from 
this class tend to be well tolerated with minimal side effects. It is important to note the potential for drug interactions with medications metabolized through the cytochrome P450 enzyme system.

Olanzapine is the latest unique agent to be added to the treatment guidelines. ${ }^{2}$ Olanzapine works on several neurotransmitters involved in the development of CINV such as dopamine, serotonin, histamine, and acetylcholine. ${ }^{4}$ Data support its role for the prevention of acute and delayed CINV as well as for the management of breakthrough CINV. Caution should be employed when using this agent in elderly patients with dementia-related psychosis as it may place them at an increased risk of death.

There are many alternative drug classes utilized for the prevention and management of CINV such as antihistamines, benzodiazepines, anticonvulsants, cannabinoids, and dopamine receptor antagonists. Medications belonging to these classes generally have lower efficacy and are associated with more adverse effects. They are also not as well studied compared to the agents mentioned above. The remainder of this review will focus on dronabinol, a member of the cannabinoid class, and its role in CINV.

\section{Pharmacology of dronabinol}

Cannabis sativa L. (also known as marijuana) contains naturally occurring delta-9-tetrahydrocannibinol (delta-9THC). The synthetic version of delta-9-THC is the active ingredient in dronabinol that makes dronabinol an orally active cannabinoid.

There are at least two types of cannabinoid receptors, CB1 and $\mathrm{CB} 2 .{ }^{5} \mathrm{CB} 1$ receptors are located throughout the central nervous system, whereas $\mathrm{CB} 2$ receptors are present on the brainstem neurons but mostly concentrated in the periphery, primarily on immunocytes and mast cells. ${ }^{6}$ These receptors can be activated not only by cannabis-derived and synthetic agonists, but also by endogenous cannabinoids produced in mammalian tissues. ${ }^{5}$ The mediating effects of dronabinol and other cannabinoids occur through these cannabinoid receptors located in neural tissues. ${ }^{7}$

Dronabinol has an onset of action of approximately $0.5-1$ hour, with a peak effect at 2-4 hours, lasting a total of 4-6 hours with the psychoactive effects. After a single dose of dronabinol, $90 \%-95 \%$ of the medication is systemically absorbed; however, only 10\%-20\% enters the systemic circulation due to the high lipid solubility and first-pass hepatic metabolism. Dronabinol has a large volume of distribution, approximately $10 \mathrm{~L} / \mathrm{kg}$, which allows for the metabolites to be released over a prolonged period of time at low levels. It undergoes first-pass hepatic metabolism, leading to active and inactive metabolites. The clearance for dronabinol varies greatly, with an average of $0.2 \mathrm{~L} / \mathrm{kg} / \mathrm{h}$. Dronabinol metabolites have been detected after a single dose more than 5 weeks after administration in the urine and feces. The major route of elimination for dronabinol is through the feces, with approximately $35 \%-50 \%$ removed by this route; however, about $10 \%-15 \%$ is found in the urine.?

\section{Evidence for clinical efficacy of dronabinol in CINV}

Dronabinol was approved by the US Food and Drug Administration (FDA) in 1985 for the treatment of CINV in patients who have failed to respond adequately to conventional antiemetic treatment.?

The endogenous cannabinoid system is an important pathway involved in the emetic response. Cannabinoids can prevent chemotherapy-induced emesis by acting at central $\mathrm{CB}_{1}$ receptors by preventing the proemetic effects of endogenous compounds such as dopamine and serotonin. ${ }^{8}$ In addition, by acting as an agonist to $\mathrm{CB}_{1}$, cannabinoids used as a treatment results in an antiemetic effect. ${ }^{9}$ Cannabinoids have been used effectively for treating CINV since $1985 .{ }^{10}$

\section{Monotherapy}

A meta-analysis evaluated a total of 1,366 patients with 30 randomized clinical trials between 1975 and $1996 .{ }^{10}$ Three different cannabinoids were evaluated among the 30 trials, with 16 trials investigating nabilone, 13 investigating dronabinol, and one investigating intramuscular levonantradol. The controls included prochlorperazine, metoclopramide, chlorpromazine, thiethylperazine, haloperidol, domperidone, alizapride, and placebo. The data showed that cannabinoids were more effective with completely controlling CINV than the active comparators or placebo in all the trials (number needed to treat $[\mathrm{NNT}]=6$ for nausea and NNT $=8$ for nausea). In patients receiving a low or highly emetogenic chemotherapy regimen, cannabinoids were similar in efficacy of complete control of CINV versus the control; however, in patients receiving moderate emetogenic risk chemotherapy regimens, cannabinoids performed better than the control.

The three cannabinoids resulted in greater adverse drug effects compared to the controls. ${ }^{10}$ There was a significant increase in the number of patients who withdrew from the studies due to intolerable adverse drug effects in 19 of the 30 trials. The increased adverse drug effects were described as being beneficial or harmful. The beneficial adverse effects 
included sensation of a "high", euphoria, drowsiness, sedation, and somnolence. The harmful adverse effects included dysphoria, depression, hallucination, and paranoia. Cannabinoids also increased the risk of arterial hypotension ( $>20 \%$ decrease in blood pressure from baseline) in patients. This meta-analysis showed that one in eleven patients would stop chemotherapy treatment if taking a cannabinoid for antiemesis compared to no patients discontinuing treatment if prescribed another antiemetic.

Rocha et al's ${ }^{11}$ meta-analysis evaluated 13 randomized clinical trials. Five trials included dronabinol, six included nabilone, and two included levonantradol. Ten of the trials used prochlorperazine as a comparator, with the remaining using alizapride, chlorpromazine, or domperidone. Dronabinol's ability for antiemetic efficacy was determined to show statistical significance over the comparator $(P=0.03$, $\mathrm{NNT}=3.4$ ). The difference in antiemetic efficacy with nabilone or levonantradol compared to controls was not statistically significant ( $P=0.21$ and $P=0.60$, respectively).

Patients in the cannabinoids group reported paranoid delusions, hallucinations, dysphoria, and depression. ${ }^{11}$ These adverse drug effects occurred exclusively in the cannabinoids group. Other adverse drug effects such as a "high" sensation, sleepiness, sedation, and euphoria occurred more frequently and more intensely in the cannabinoids group. However, only $30 \%$ of the 400 patient dropouts were due to toxicities.

\section{Dronabinol in combination with ondansetron}

Meiri et $\mathrm{al}^{8}$ conducted a randomized, double-blind, placebocontrolled, parallel group trial that enrolled 64 patients receiving moderate or highly emetic chemotherapy. ${ }^{8}$ The objective of this study was to determine if 4 days after chemotherapy, adding dronabinol to a prophylactic regimen for acute CINV and continuing treatment either alone or in combination with ondansetron can help prevent delayed CINV. The four parallel groups were dronabinol monotherapy ( $D ; n=17)$, ondansetron monotherapy $(\mathrm{O} ; \mathrm{n}=17)$, combination with dronabinol and ondansetron $(D+O ; n=16)$, and placebo $(n=14)$. Patients in all four groups received dexamethasone $20 \mathrm{mg}$ PO (by mouth) and ondansetron $16 \mathrm{mg}$ intravenous prechemotherapy on day 1 . In the three active treatment groups, patients also received dronabinol $2.5 \mathrm{mg}$ PO both prechemotherapy and postchemotherapy on day 1 . Day 2 consisted of fixed doses with dronabinol $2.5 \mathrm{mg}$ PO four times daily and/or ondansetron $8 \mathrm{mg}$ PO twice daily in the respective groups. Days 3-5 were flexible dosing days, with patients being allowed to take dronabinol 2.5-5 mg PO four times a day and/or ondansetron 4-8 mg PO twice daily depending on tolerability. In the placebo group, a placebo was matched with the doses of dronabinol and/or ondansetron. In all groups, patients were provided with rescue medications consisting of metoclopramide PO, prochlorperazine $\mathrm{PO}$, and prochlorperazine suppository to be used on days 1-8 for intolerable nausea and vomiting or retching after the maximum prescribed study doses.

In this trial, there were more females (37 out of 61 patients, 61\%) compared to males (24 out of 61 patients, $39 \%) .{ }^{8}$ The two most common cancer diagnoses were breast cancer ( 26 out of 64 patients, $41 \%$ ) and non-small-cell lung cancer (14 out of 64 patients, 22\%). Among all four groups, 29 patients $(45 \%)$ took all of the appropriate study medications in the correct dosages over the 5-day trial period. A total response was defined as no vomiting and/or retching, intensity of nausea less than $5 \mathrm{~mm}$ on a $100 \mathrm{~mm}$ visual analog scale (VAS), and no rescue medications. VAS is ranked from 0 to $100 \mathrm{~mm}$, with $0 \mathrm{~mm}$ meaning no nausea and $100 \mathrm{~mm}$ meaning intractable nausea. For day 1 results, the three active treatment groups were combined and compared to the placebo group. The total response during active treatment on day 1 for the combined active treatment (CAT) group was 79\% compared to $40 \%$ for the placebo group. For days $2-5$, the total response for the $\mathrm{D}, \mathrm{O}, \mathrm{D}+\mathrm{O}$, and placebo groups were $54 \%, 58 \%, 47 \%$, and $20 \%$, respectively. The percentage of patients without nausea on day 1 in CAT group was 79\% and in the placebo group 38\%. For days $2-5$, the percentages were $71 \%, 64 \%, 53 \%$, and $15 \%$ in the D, O, D + O, and placebo groups, respectively. The reported VAS for nausea intensity was 7.65 in the CAT group and 30.67 in the placebo group. For days 2-5, the VAS was 10.1 in the D group, 24 in the O group, 14.3 in the $\mathrm{D}+\mathrm{O}$ group, and 48.4 in the placebo group. Overall, the complete response rate was $62 \%$ in the $\mathrm{D}$ group, $58 \%$ in the $\mathrm{O}$ group, $60 \%$ in the $\mathrm{D}+\mathrm{O}$ group, and $20 \%$ in the placebo group. In addition, the active treatment groups reduced the number of vomiting episodes to zero and decreased the duration of vomiting and retching to 0 hours by days 4 and 5 . The duration of nausea was comparable between all four groups. On the flexible dosage days, the median dosage in the D group was $20 \mathrm{mg} / \mathrm{d}$ of dronabinol, $16 \mathrm{mg} / \mathrm{d}$ of ondansetron in the $\mathrm{O}$ group, and $17.5-20 \mathrm{mg} / \mathrm{d}$ of dronabinol and 12-16 mg/d of ondansetron in the $\mathrm{D}+\mathrm{O}$ group. Rescue medications were used in all four groups $(24 \%$ in $\mathrm{D}, 31 \%$ in $\mathrm{O}, 12 \%$ in $\mathrm{D}+\mathrm{O}$, and $43 \%$ in placebo groups).

Treatment-emergent adverse drug effects were reported in all four groups, with the largest percentage in the ondansetron group. ${ }^{8}$ Adverse drug effects were reported in $82 \%$ in 
the $\mathrm{D}$ group, $88 \%$ in the $\mathrm{O}$ group, $71 \%$ in the $\mathrm{D}+\mathrm{O}$ group, and $50 \%$ in the placebo group. The percentage of patients who permanently discontinued a study medication because of a treatment-emergent adverse drug effect was $6 \%$ in the $\mathrm{D}$ group, $13 \%$ in the $\mathrm{O}$ group, $18 \%$ in the $\mathrm{D}+\mathrm{O}$ group, and no patients in the placebo group.

\section{Dronabinol in combination with prochlorperazine}

Lane et $\mathrm{al}^{12}$ conducted a multicentered, randomized, parallel group, double-blind trial that enrolled 62 patients. All the patients had previously received chemotherapy and antiemetics. Patients were eligible for the trial if they were receiving any chemotherapy agents except high-dose cisplatin $\left(>60 \mathrm{mg} / \mathrm{m}^{2}\right)$. The most common chemotherapy agents were doxorubicin and cyclophosphamide $(n=26), 5$-fluorouracil $(n=14)$, vincristine $(n=13)$, and etoposide $(n=10)$. Two or three drug chemotherapy regimens were given to $79 \%$ of patients. Patients were randomized to one of three arms: dronabinol 10 mg PO every 6 hours with placebo $(n=21)$, prochlorperazine $10 \mathrm{mg}$ PO every 6 hours with placebo $(\mathrm{n}=21)$, or dronabinol $10 \mathrm{mg}$ PO every 6 hours with prochlorperazine $10 \mathrm{mg}$ PO every 6 hours $(n=20)$. Patients started the prescribed antiemetic 24 hours prior to starting chemotherapy and continued it until 24 hours after the last dose of chemotherapy.

The overall complete response rate was defined as no episodes of nausea or vomiting and occurred in $41 \%$ in the dronabinol group and $30 \%$ in the prochlorperazine group $(P<0.0001) .{ }^{12}$ No complaints of nausea or vomiting occurred in $41 \%$ in the dronabinol group, $30 \%$ in the prochlorperazine group, and $47 \%$ in the combination group. Two or fewer nausea or vomiting episodes occurred in $71 \%, 45 \%$, and $65 \%$ in the dronabinol, prochlorperazine, and combination groups, respectively. The median duration per episode of nausea or vomiting was 2 minutes in the combination group compared to 5 minutes in the dronabinol and prochlorperazine groups $(P<0.001)$. In addition, the severity of the nausea was less in the combination group versus the dronabinol and prochlorperazine groups $(P<0.001)$. The duration per episode of nausea or vomiting in the dronabinol group was 2 and 10 minutes versus 4 and 15 minutes in the prochlorperazine group. However, the total duration of nausea and vomiting episodes did not differ between the three treatment groups. Eleven patients reported anticipatory nausea (30\% in dronabinol group, $0 \%$ in the prochlorperazine group, and $26 \%$ in the combination group).

Thirty-four patients reported adverse drug effects in the dronabinol $(n=16)$, prochlorperazine $(n=7)$, and combination
( $n=11)$ groups. $^{12}$ The difference of reported adverse drug effects was statistically significant between the dronabinol and prochlorperazine groups $(P<0.01)$. Most of the adverse drug effects reported were mild to moderate. The most common type of adverse effects reported were neuropsychotropic, seen in a total of $48 \%$ of patients. The incidence of neuropsychotropic adverse drug effects that was reported in the dronabinol and prochlorperazine groups was $62 \%$ versus $29 \%(P=0.06)$. In the combination group, it was reported in $55 \%$ of patients. Overall, 14 patients withdrew (ten patients in dronabinol group and four in combination group) due to adverse drug effects, with neuropsychotropic effects being the most common reason and beginning during the prechemotherapy phase.

\section{Indicated dronabinol dosage}

Dronabinol is typically prescribed at a dosage of $5 \mathrm{mg}$ PO three or four times daily to control CINV. ${ }^{7}$ On the basis of the patient's response after each chemotherapy cycle, the dose may be increased or decreased as tolerated. Another option for dosing dronabinol is $5 \mathrm{mg} / \mathrm{m}^{2}$ PO every $1-3$ hours prechemotherapy and then every 2-4 hours for a total of 4-6 doses/d. The maximum individual dose is $15 \mathrm{mg} / \mathrm{m}^{2}$.

\section{Patient perspectives of dronabinol}

$\mathrm{CB}_{1} / \mathrm{CB}_{2}$ receptor agonists can produce adverse effects in patients, and many of these are likely caused by the activation of central $\mathrm{CB}_{1}$ receptors rather than $\mathrm{CB}_{2}$ or peripheral $\mathrm{CB}_{1}$ receptors. $^{5}$

In 18 cross-over trials included in the Tramer et $\mathrm{al}^{10}$ metaanalysis, when patients were questioned which antiemetic was preferred, $38 \%-90 \%$ of patients preferred cannabinoids. Four trials compared cannabinoids to placebo. Out of 202 patients, 153 patients preferred cannabinoids versus 27 patients preferring the placebo $(\mathrm{NNT}=1.6)$. In ten additional trials comparing cannabinoids to an active control, 371 out of $604(61 \%)$ preferred cannabinoids versus 156 patients (26\%) preferring the active control.

In the Rocha et al's ${ }^{11}$ meta-analysis, 18 double-blind and cross-over trials $(n=1,138)$ included an analysis of the patient's preference of cannabinoids, and it resulted in a statistically significant difference in favor of cannabinoids (NNT $=1.8, P<0.00001)$. The cannabinoids included in the different trials included dronabinol, nabilone, and levonantradol compared to the controls of prochlorperazine, chlorpromazine, domperidone, haloperidol, alizapride, metoclopramide, and placebo. A relationship was determined with the control drug that was utilized. 
It is suggested that patients prefer the "beneficial" adverse drug effects associated with dronabinol, such as, sedation, sensation of a "high", and somnolence. ${ }^{10,11}$ These adverse drug effects might provide relief while receiving chemotherapy. ${ }^{11}$ Another view is since CINV has such a major impact on the patient's QoL and can cause such discomfort to the patient, the patients prefer cannabinoids' adverse drug effects instead of the conventional medications that might be less effective in preventing and relieving the CINV. ${ }^{11}$

\section{Medical marijuana}

Many states have legalized marijuana for medical use, including the condition of cancer. ${ }^{13}$ The legal limit of the amount allowed for a patient to possess varies by state. Technically marijuana contains more than 60 pharmacologically active cannabinoids, with the primary active cannabinoids being THC and cannabidiol. The therapeutic effect depends on the concentration of THC in addition to the THC-to-cannabidiol ratio. Various strains of marijuana are engineered to have different concentrations and ratios to achieve desired pharmacologic effects. Medical marijuana may be purchased from dispensaries in various dosage forms as well, and is most commonly dispensed in the form used to smoke as "cigarettes" or with a water pipe. ${ }^{14}$ It may also be inhaled through a vaporizer, eaten in food, or applied topically as a lotion.

It is important to note that adverse effects on the cardiovascular, respiratory, and central nervous system have been associated with marijuana use. Marijuana smoke has been reported to contain more carcinogens than cigarette smoke, and may lead to head and neck cancer, lung cancer, as well as bronchitis. ${ }^{9}$ The development of atrial fibrillation, myocardial infarction, stroke, drowsiness, dizziness, nightmares, difficulty sleeping, acute toxic psychosis, anxiety, and depression among many other adverse effects has been associated with marijuana use. ${ }^{914}$ Another concern in cancer patients is the reported immunosuppressive properties of marijuana.

Few studies have evaluated medical marijuana alone or in combination to treat nausea and vomiting related to chemotherapy. The published studies that have been conducted have mixed results. One study of 15 patients receiving adjuvant high-dose methotrexate for sarcoma were randomized to three paired trials of placebo-THC or THC-placebo with the patients serving as their own control..$^{15} \mathrm{THC}$ was administered as capsules and cigarettes or matching placebos. Fourteen of the 15 patients reported a reduction in nausea and vomiting when using THC. Another study conducted by the same group in eight patients receiving adjuvant doxorubicin and cyclophosphamide for sarcoma were randomized to THC-placebo or placebo-THC in paired trials to serve as their own control. ${ }^{16} \mathrm{THC}$ was administered as oral capsules and cigarettes compared to matching placebos. Although the investigators used the same trial design and THC regimen, a beneficial effect of THC was not seen in this patient population. The lack of benefit in this trial is thought to potentially be from lower THC concentrations achieved in this group of patients and presence of anticipatory nausea and vomiting in half of the patients.

The largest account of patients receiving marijuana for CINV was published by Musty and Rossi in 2001. ${ }^{17}$ This group compiled a report of state-run clinical trials that had been conducted evaluating Cannabis sativa (smoked marijuana and/or delta-9-tetrahydrocannabinol capsules) efficacy in reducing CINV. Six trials were included in the analysis comprising 748 patients who smoked marijuana prior to and/ or after chemotherapy and 345 patients who used oral THC capsules. For the patients who smoked marijuana, $70 \%-100 \%$ experienced relief from nausea and vomiting, and $76 \%-88 \%$ of those who ingested the oral THC capsules experienced relief. Short-term side effects reported included sedation, a "high", and smoke intolerance. More studies are needed in this area to evaluate the efficacy and safety as additional states approve medical marijuana and more patients gain access to this management option.

\section{Future directions}

Dronabinol is utilized for breakthrough CINV based upon available evidence already discussed and recommendations by major oncology guidelines. Dronabinol is currently being investigated in a few studies for additional roles in the management of CINV. A completed, not published study has investigated the use of dronabinol versus standard ondansetron antiemetic therapy in the prevention of delayed CINV or retching after moderate-to-high emetogenic chemotherapy. ${ }^{18}$ Patients were randomized to ondansetron, dronabinol, combination therapy, or placebo. The study outcome was to measure response of nausea and vomiting/retching, intensity, and use of rescue medication. An additional study completed, but yet to be published, has investigated palonosetron and dexamethasone with or without dronabinol in the prevention of CINV with moderately emetogenic chemotherapy. ${ }^{19}$ Patients were assessed for protection against the development of vomiting, nausea, and use of rescue therapy. In an ongoing study of patients with primary glioma receiving chemotherapy, dronabinol is being administered to assess its tolerability, toxicity, and impact on QoL. ${ }^{20}$ Assessment 
of emesis will be completed by patients filling out a daily appetite and nausea/vomiting log as well as a QoL functional living index emesis scale.

With medical marijuana remaining a schedule I substance on a federal level, no studies are registered with clnicaltrials.gov to investigate its role in the management of CINV. Anecdotally patients report benefit, but more research is needed to identify the most appropriate dose, dosage form, drug-drug interactions, and safety concerns with its use before a role for medical marijuana can be elucidated in the management of CINV.

\section{Conclusion}

Current guidelines as well as the FDA-approved indications consider dronabinol's role to be in the management of breakthrough CINV. Dronabinol has a unique mechanism of action and adverse effect profile that should be considered when treating a patient with this medication. Unfortunately, there are few ongoing studies evaluating the role of dronabinol in the management of CINV. Two completed, yet unpublished, studies have evaluated dronabinol in combination with a $5-\mathrm{HT}_{3} \mathrm{RA}$ as a prophylactic strategy. At this time, there is insufficient data to support the routine use of dronabinol as an antiemetic in all chemotherapeutic regimens. Data do support the beneficial effects of dronabinol in the breakthrough CINV setting. Further study of the scope of dronabinol's potential efficacy is warranted.

\section{Disclosure}

The authors report no conflicts of interest in this work.

\section{References}

1. Fernandez-Ortega P, Caloto MT, Chirveches E, et al. Chemotherapyinduced nausea and vomiting in clinical practice: impact on patient's quality of life. Support Care Cancer. 2012;20:3141-3148.

2. National Comprehensive Cancer Network. NCCN Clinical Practice Guidelines in Oncology (NCCN Guidelines) ®: Antiemesis Version 2.2015. Fort Washington, PA: National Comprehensive Cancer Network; 2015.

3. Haiderali A, Menditto L, Good M, Teitelbaum A, Wegner J. Impact on daily functioning and indirect/direct costs associated with chemotherapyinduced nausea and vomiting (CINV) in a US population. Support Care Cancer. 2011;19:843-851.
4. Natale J. Reviewing current and emerging antiemetics for chemotherapy-induced nausea and vomiting prophylaxis. Hosp Pract. 2015;43(4):226-234.

5. Pertwee RG. Emerging strategies for exploiting cannabinoid receptor agonists as medicines. Br J Pharmacol. 2009;156:397-411.

6. Slatkin NE. Cannabinoids in the treatment of chemotherapy-induced nausea and vomiting: beyond prevention of acute emesis. J Support Oncol. 2007;5(Suppl 3):1-9.

7. Marinol(R) [package insert]. High Point, NC: Unimed Pharmaceuticals, Inc; 2004.

8. Meiri E, Jhangiani H, Vredenburgh, et al. Efficacy of dronabinol alone and in combination with ondansetron versus ondansetrom alone for delayed chemotherapy-induced nausea and vomiting. Curr Med Res Opin. 2007;23(3):533-543.

9. Todaro B. Cannabinoids in the treatment of chemotherapy-induced nausea and vomiting. JNCCN. 2012;10(4):487-492.

10. Tramer MR, Carroll D, Campbell FA, Reynolds JM, Moore RA, McQuay HJ. Cannabinoids for control of chemotherapy induced nausea and vomiting: quantitative systemic review. BMJ. 2001;323:16-21.

11. Rocha FM, Stefano SC, De Cassia Haiek R, Oliveira LR, Silveira DD. Therapeutic use of Cannabis sativa on chemotherapy-induced nausea and vomiting among cancer patients: systematic review and metaanalysis. Eur J Cancer Care. 2008;17:431-443.

12. Lane M, Vogel CL, Ferguson J, et al. Dronabinol and prochlorperazine in combination for treatment of cancer chemotherapy-induced nausea and vomiting. J Pain Symptom Manage. 1991;6(6):352-359.

13. Hill KP. Medical marijuana for treatment of chronic pain and other medical and psychiatric problems. A clinical review. JAMA. 2015;313(24):2474-2483.

14. Seamon MJ, Fass JA, Maniscalco-Feichtl M, Abu-Shraie NA. Medical marijuana and the developing role of the pharmacist. Am J Health Syst Pharm. 2007;64:1037-1044.

15. Chang AE, Shiling DJ, Stillman RC, et al. Delta-9-tetrahydrocannabinol as an antiemetic in cancer patients receiving high-dose methotrexate. A prospective, randomized evaluation. Ann Intern Med. 1979;91:819-824.

16. Chang AE, Shiling DJ, Stillman RC, et al. A prospective evaluation of delta-9-tetrahydrocannabinol as an antiemetic in patients receiving adriamycin and cytoxan chemotherapy. Cancer. 1981;47:1746-1751.

17. Musty RE, Rossi, R. Effects of smoked cannabis and oral $\Delta 9$-trahydrocannabinol on nausea and emesis after cancer chemotherapy: A review of state clinical trials. J Cannabis Therapeutics. 2001;1(1):29-56.

18. Solvay Pharmaceuticals. Dronabinol versus standard ondansetron antiemetic therapy in preventing delayed-onset chemotherapy-induced nausea and vomiting. NLM identifier: NCT00642512. Available from: https:// clinicaltrials.gov/ct2/show/NCT00642512. Accessed April 13, 2016.

19. National Cancer Institute and Solvay Pharmaceuticals; M.D. Anderson Cancer Center. Palonosetron and dexamethasone with or without dronabinol in preventing nausea and vomiting in patients receiving chemotherapy for cancer. NLM Identifier: NCT00553059. Available from: https:// clinicaltrials.gov/ct2/show/NCT00553059 . Accessed April 13, 2016.

20. Solvay Pharmaceuticals; Duke University. A pilot study of dronabinol for adult patients with primary gliomas. NLM Identifier: NCT00314808. Available from: http://clinicaltrials.gov/show/NCT00314808. Accessed April 13, 2016.
Cancer Management and Research

\section{Publish your work in this journal}

Cancer Management and Research is an international, peer-reviewed open access journal focusing on cancer research and the optimal use of preventative and integrated treatment interventions to achieve improved outcomes, enhanced survival and quality of life for the cancer patient. The manuscript management system is completely online and includes

\section{Dovepress}

a very quick and fair peer-review system, which is all easy to use. Visit $\mathrm{http}: / / \mathrm{www}$.dovepress.com/testimonials.php to read real quotes from published authors. 